\title{
Efeito do comprimento do corpo de prova nas propriedades mecânicas do carvão de Eucalyptus
}

Taís Regina Lima Abreu Veiga ${ }^{1 *}$, José Tarcísio Lima ${ }^{1}$, Thiago Campos Monteiro², Maria Fernanda Vieira Rocha ${ }^{1}$, Márcia Silva de Jesus ${ }^{1}$, Selma Lopes Goulart ${ }^{3}$

\author{
${ }^{1}$ Universidade Federal de Lavras, Campus Universitário, C P 3037, CEP 37200-000, Lavras, MG, Brasil \\ ${ }^{2}$ Universidade Federal de Minas Gerais, Av. Universitária, 1000, CEP 39404-547, Montes Claros, MG, Brasil \\ ${ }^{3}$ Universidade Federal Rural da Amazônia, AC Parauapebas, Cidade Nova, C P 3017, CEP 68515-970, Parauapebas, PA , Brasil
}

"Autor correspondente:

tr.abreu@hotmail.com

Termos para indexação:

Madeira

Resistência

Qualidade do carvão

Index terms:

Wood

Strength

Charcoal quality

Histórico do artigo:

Recebido em 08/10/2015

Aprovado em 02/12/2016

Publicado em 30/12/2016

doi: 10.4336/2016.pfb.36.88.1073
Resumo - As propriedades mecânicas do carvão são indicadoras de qualidade e influenciam no processo siderúrgico. Os ensaios de avaliação (queda e tamboramento), em geral, apresentam resultados variáveis, comprometendo sua confiabilidade. Por isso, é importante desenvolver metodologias que produzam resultados comparáveis. Esse trabalho objetivou analisar a influência do comprimento do corpo de prova na determinação da rigidez e resistência da madeira e do carvão. Foram utilizados clones de Eucalyptus urophylla, dos quais foram produzidos corpos de prova de seção transversal de $25 \mathrm{~mm}$ x $25 \mathrm{~mm}$ e comprimentos de $50 \mathrm{~mm}, 75 \mathrm{~mm}, 100 \mathrm{~mm}$ e $125 \mathrm{~mm}$. Dez amostras de cada comprimento foram selecionadas para a realização do ensaio com madeira, e outras dez para os ensaios com carvão. O módulo de elasticidade e a resistência à compressão foram determinados em ensaio de compressão paralela às fibras. Houve efeito significativo do comprimento do corpo de prova sobre a rigidez e resistência da madeira e do carvão, sendo que os melhores resultados foram encontrados para as dimensões $25 \mathrm{~mm}$ x $25 \mathrm{~mm}$ x $75 \mathrm{~mm}$. Apenas a dimensão de $25 \mathrm{~mm}$ x $25 \mathrm{~mm}$ x $50 \mathrm{~mm}$ se mostrou inadequada, apresentando resultados inferiores aos relatados em literatura.

\section{Effect of the sample length in compressive strength of Eucalyptus wood and charcoal}

\begin{abstract}
Mechanical properties of charcoal are indicators of quality and they influence in steelmaking process. In general, assessment tests (falling and tumbling) present variable results compromising their reliability. So, it is important to develop methodologies that produce comparable results. This study aimed to analyze the influence of sample length to determine stiffness and strength of wood and charcoal. Samples were produced from Eucalyptus urophylla clones, presenting cross section $25 \mathrm{~mm} \times 25 \mathrm{~mm}$ and lengths of $50 \mathrm{~mm}, 75 \mathrm{~mm}, 100 \mathrm{~mm}$ and $125 \mathrm{~mm}$. Ten samples of each length were selected for testing wood, and another ten for testing charcoal. The modulus of elasticity and compressive strength were determined in compression test parallel to the fibers. There was a significant effect of sample length on stiffness and strength of wood and charcoal. The best results were found for samples of $25 \mathrm{~mm} \mathrm{x}$ $25 \mathrm{~mm} \times 75 \mathrm{~mm}$. Only the dimension $25 \mathrm{~mm}$ x $25 \mathrm{~mm}$ x $50 \mathrm{~mm}$ proved inadequate, presenting lower results than those reported in literature.
\end{abstract}




\section{Introdução}

O carvão vegetal é um produto da transformação térmica da madeira, que pode ser utilizado não apenas na cocção de alimentos, mas também em processos industriais, como na produção do aço e de gás. No Brasil, constitui-se em uma matéria prima de grande importância para o setor siderúrgico, uma vez que fornece energia e gás redutor para o minério de ferro. Atualmente, aproximadamente $86 \%$ do carvão vegetal produzido no país são destinados ao setor siderúrgico. Nos últimos anos, o Brasil produziu, em média, 32 milhões de $\mathrm{t}$ de ferro gusa, sendo que cerca de $30 \%$ da produção foram obtidos a partir da utilização desse insumo como agente redutor do minério (Indústria Brasileira de Árvores, 2015).

Para a utilização do carvão vegetal como termorredutor é essencial caracterizá-lo, uma vez que suas propriedades mecânicas estão diretamente ligadas à qualidade final do aço produzido. Segundo dados da Fundação Centro Tecnológico de Minas Gerais (Cetec) (1980), quanto maior for sua resistência à compressão e rigidez, maior será a sua capacidade de sustentação do leito no interior do alto forno siderúrgico.

Dentre as propriedades desejáveis do carvão vegetal para siderurgia podem-se citar a composição química (Brito, 1993; Vale et al., 2011), densidade elevada (Gomes \& Oliveira, 1980), tamanho médio das partículas e friabilidade (Mendes et al., 1982), que estão ligadas à resistência mecânica do carvão, fator importante no processo de fabricação do aço.

Na literatura há muitos estudos sobre os parâmetros físicos e químicos de qualidade do carvão (Neves et al., 2011; Reis et al., 2012a, 2012b; Protásio et al., 2013, 2014a, 2014b, 2014c). Porém, pouco se sabe sobre as propriedades mecânicas dos mesmos e sobre normativas que descrevam e padronizem os ensaios mecânicos.

Atualmente, o setor siderúrgico aplica testes tradicionais, como o de tamboramento e de queda, que permitem obter uma indicação da friabilidade do carvão vegetal. Porém, tais testes não caracterizam individualmente a peça carbonizada e não garantem resultados precisos. Com a busca constante e necessária da autossuficiência, as empresas estão dando importância ao desenvolvimento de novas tecnologias de produção de madeira e, principalmente, avaliação de sua qualidade, bem como da transformação do carvão vegetal (Trugilho et al., 2001).
Diante da ausência de métodos e da dificuldade em se avaliar a resistência mecânica do carvão vegetal em peças individuais, é importante desenvolver um método que vise à avaliação confiável do "material carvão" e de suas propriedades mecânicas para melhor caracterizar a matéria-prima que a indústria siderúrgica necessita. Dessa forma, o objetivo do presente trabalho foi analisar a influência dos comprimentos dos corpos de prova na determinação da rigidez e resistência à compressão paralela às fibras da madeira e do carvão, por meio de ensaios destrutivos. Registra-se ainda que, embora o trabalho focalize as propriedades mecânicas do carvão vegetal, são apresentadas, também, caracterizações mecânicas da madeira, como um parâmetro de comparação e de possíveis correlações.

\section{Material e métodos}

Para a realização do trabalho foram utilizadas madeiras de clones de Eucalyptus urophylla, plantados com espaçamento de 3,0 $\mathrm{m} \times 3,0 \mathrm{~m}$, no município de Paraopeba, MG. Esses clones tinham sete anos de idade e eram provenientes de plantios comerciais da empresa Vallourec Florestal Ltda.

As árvores foram abatidas e seccionadas em toras de 1,5 $\mathrm{m}$ de comprimento, que posteriormente foram desdobradas em pranchões de 2,5 cm de espessura. Desses pranchões foram retirados corpos de prova de seção transversal de $25 \mathrm{~mm}$ x $25 \mathrm{~mm}$ e comprimentos equivalentes a $50 \mathrm{~mm}, 75 \mathrm{~mm}, 100 \mathrm{~mm}$ e $125 \mathrm{~mm}$, para a realização dos ensaios de compressão paralela às fibras. Vinte corpos de prova de cada comprimento foram selecionados para a realização do ensaio mecânico, sendo metade correspondente aos ensaios com madeira e o restante destinado à carbonização e, posteriormente, determinação da rigidez e resistência do carvão.

$\mathrm{O}$ módulo de elasticidade (MOE) e a resistência à compressão (RC) tanto da madeira como do carvão foram determinados em ensaio de compressão paralela às fibras com base na norma ASTM-D 143-94 (American Society for Testing and Materials, 2000). Diante da ausência de uma metodologia específica para determinar as propriedades mecânicas do carvão vegetal, adotouse as diretrizes seguidas por Vieira (2009), onde a velocidade de aplicação da carga foi de $0,05 \mathrm{~mm} \mathrm{~min}^{-1}$ e o ponto de interrupção do ensaio estipulado em $20 \%$ de perda de resistência do material. 
As carbonizações foram realizadas em forno elétrico adaptado com sistema de recuperação de gases, onde a temperatura inicial foi sempre igual a $100{ }^{\circ} \mathrm{C}$ e a temperatura final máxima foi $450{ }^{\circ} \mathrm{C}$, permanecendo estabilizada por um período de $30 \mathrm{~min}$. A taxa de aquecimento foi fixada em $0,5{ }^{\circ} \mathrm{C} \mathrm{min}^{-1}$, com base nos estudos realizados por Vieira (2009), que avaliando diferentes taxas de carbonização constatou que essa velocidade resultou em corpos de prova com menos defeitos.

Os resultados obtidos foram submetidos à análise de variância (Anova) e no caso de efeitos significativos foram efetuadas comparações de médias por teste de Scott- Knott em nível de 5\% de probabilidade de erro.

\section{Resultados e discussão}

Os valores médios dos módulos de elasticidade (MOE) da madeira e do carvão vegetal, em função do comprimento dos corpos de prova, estão apresentados na Figura 1. Observa-se que houve efeito significativo do comprimento do corpo de prova sobre a rigidez dos materiais.

De modo geral, o módulo de elasticidade da madeira diminui com a redução do comprimento do corpo de prova. Porém, tal efeito torna-se menos expressivo com a carbonização do material, pois apenas os carvões de $50 \mathrm{~mm}$ de comprimento apresentaram rigidez estatisticamente inferior (Figura 1).

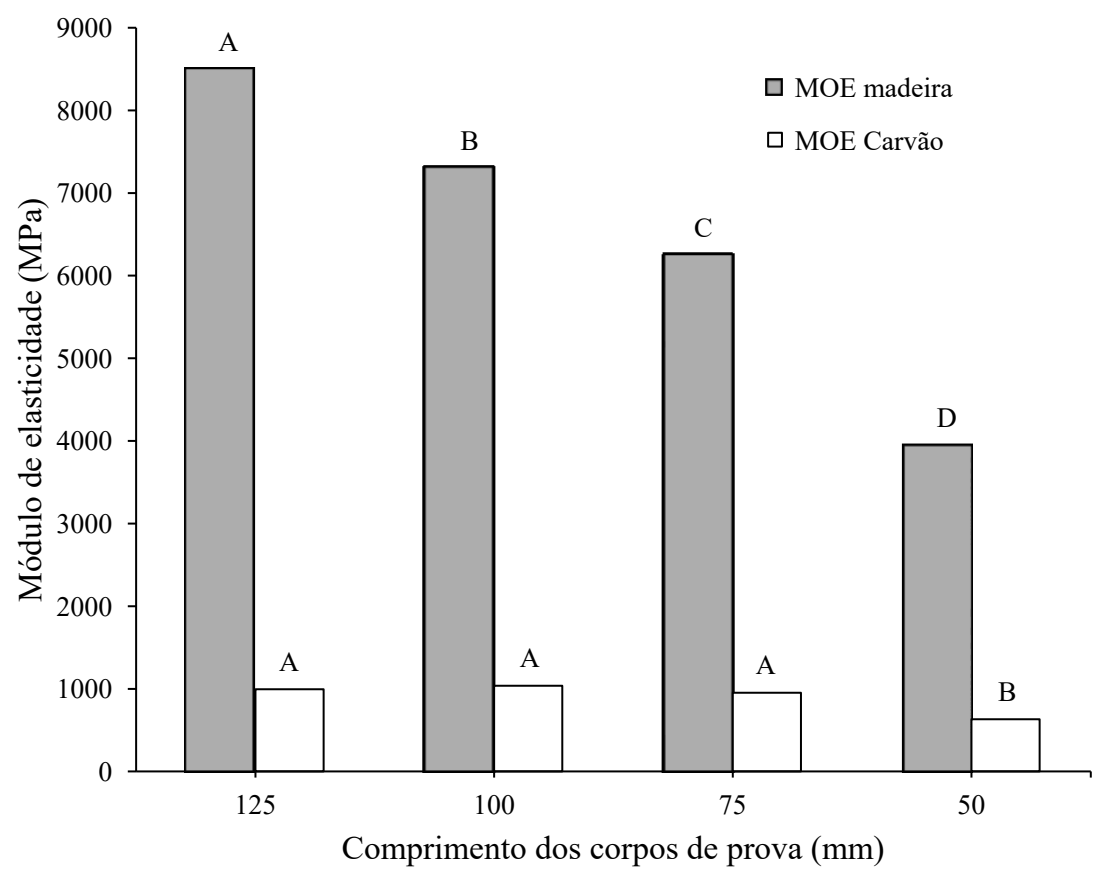

Figura 1. Valores médios de módulo de elasticidade (MOE) da madeira e do carvão em função do comprimento dos copos de prova. As médias seguidas de mesma letra para cada material testado não diferem entre si pelo teste ScottKnott $(\mathrm{P}<0,05)$.

Os valores de módulo de elasticidade da madeira estão coerentes com aqueles apresentados por Cruz et al. (2003) e Hein et al. (2009), que estudando madeira de Eucalyptus relataram valores variando de 6590 a $8993 \mathrm{MPa}$. No entanto, foram superiores aos resultados relatados por Moutinho (2013) e Couto et al. (2015). Moutinho (2013) estudou carvão vegetal produzido de madeiras de Eucalyptus com idades entre cinco e seis anos, e temperatura final de carbonização igual a $400{ }^{\circ} \mathrm{C}$, e encontrou valores de resistência à compressão do carvão variando de 7,32 a 15,69 MPa, e módulo de elasticidade variando de $316 \mathrm{MPa}$ e $737 \mathrm{MPa}$.

Em média, a redução de $25 \mathrm{~mm}$ no comprimento dos corpos de prova resultou em queda de $14 \%$ no $\mathrm{MOE}$ da madeira, exceto na dimensão de $25 \mathrm{~mm}$ x $25 \mathrm{~mm}$ x $50 \mathrm{~mm}$, onde se observou redução de $37 \%$ do MOE. De acordo com a NBR 7190 (Associação Brasileira de Normas Técnicas, 1997) o comprimento do corpo de prova deve ser no minimo três vezes maior que a sua largura, para que não haja prejuizos nas propriedades 
mecânicas. Dessa forma, nota-se que a dimensão 25 x 25 x $50 \mathrm{~mm}$ não respeita a proporção estabelicida, o que provavelmente ocasionou resultados inferiores aos relatados na literatura (Cruz et al., 2003; Hein et al., 2009).

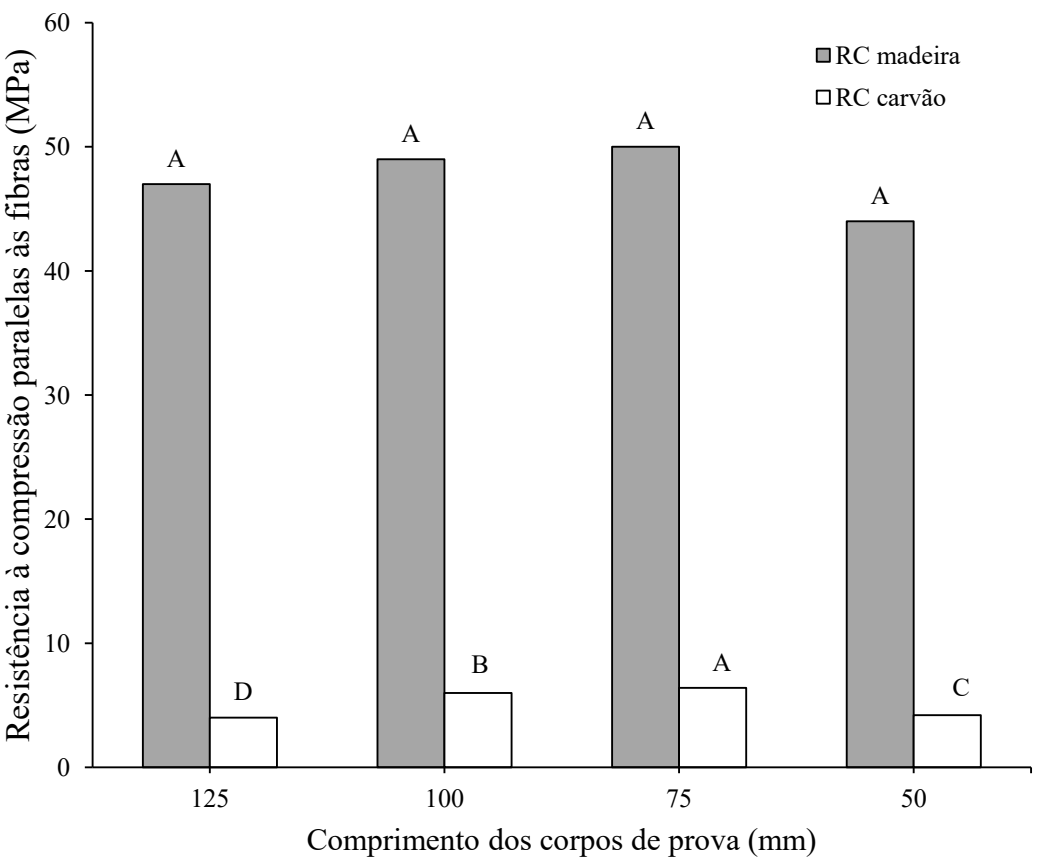

Figura 2. Valores médios de resistência à compressão paralela às fibras da madeira e do carvão em função da dimensão dos copos de prova. As médias seguidas de mesma letra para cada material testado não diferem entre si pelo teste Scott-Knott $(\mathrm{P}<0,05)$.

Observa-se na Figura 2 que não houve efeito significativo do comprimento dos corpos de prova sobre a resistência à compressão $(\mathrm{RC})$ da madeira, diferentemente do carvão vegetal, onde se nota aumento da RC nos comprimento de $100 \mathrm{~mm}$ e $75 \mathrm{~mm}$ e redução nas dimensões de $25 \mathrm{~mm}$ x $25 \mathrm{~mm}$ x $50 \mathrm{~mm}$. Semelhante ao observado para o módulo de elasticidade, apenas os corpos de prova de dimensão $25 \mathrm{~mm}$ x $25 \mathrm{~mm}$ x $50 \mathrm{~mm}$ apresentaram valores de resistência à compressão da madeira inferiores aos da literatura (Moura, 2000; Cruz et al., 2003; Hein et al., 2009; Moutinho, 2013). Em geral, os resultados foram semelhantes aos relatados por Araujo (1952) e Vieira (2009).

Comparando-se as propriedades mecânicas da madeira e do carvão, observar-se que o módulo de elasticidade à compressão paralela às fibras do carvão foi 8 vezes menor (Figura 1) enquanto que a resistência à compressão foi 9 vezes menor (Figura 2). Isso indica que a carbonização reduz a rigidez e a resistência,
O comportamento da resistência à compressão paralela às fibras da madeira e do carvão vegetal em relação ao comprimento dos corpos de prova foi heterogêneo (Figura 2). 
lado, o comprimento da madeira não tem essa mesma relação com a RC (Figura 2).

\section{Conclusões}

Houve efeito significativo do comprimento do corpo de prova sobre a rigidez e a resistência da madeira e do carvão. Observou-se que a rigidez do carvão foi estatisticamente igual para todas as dimensões, exceto para as amostras de $25 \mathrm{~mm} \times 25 \mathrm{~mm} \times 50 \mathrm{~mm}$, que apresentaram valores de módulo de elasticidade muito inferior, mostrando-se inadequada para a realização do ensaio.

O comportamento da resistência à compressão paralela às fibras da madeira e do carvão vegetal em relação ao comprimento dos corpos de prova foi heterogêneo. Os maiores valores foram encontrados para a dimensão de $25 \mathrm{~mm} \times 25 \mathrm{~mm} \times 75 \mathrm{~mm}$, sendo, portanto, a mais indicada para a realização dos ensaios.

\section{Referências}

American Society for Testing and Materials. D 143-94: standard test methods for small clear specimens of timber. Philadelphia, 2000. $31 \mathrm{p}$.

Associação Brasileira de Normas Técnicas. NBR 7190: projeto de estruturas de madeira. Rio de Janeiro, 1997. 107 p.

Araujo, J. B. Carvão vegetal para alto-fornos. Rio de Janeiro: Ministério da Agricultura, Departamento Nacional da Produção Mineral, Laboratório da Produção Mineral, 1952. 135 p.

Brito, J. O. Reflexões sobre a qualidade do carvão vegetal para uso siderúrgico. Piracicaba: IPEF, 1993. 6 p. (Circular técnica, 181).

Couto, A. M. et al. Qualidade do carvão vegetal de Eucalyptus e Corymbia produzido em diferentes temperaturas finais de carbonização. Scientia Forestalis, v. 43, n. 108, p. 817-831, 2015. DOI: $10.18671 /$ scifor.v43n108.7.

Cruz, C. R. et al. Variações dentro das árvores e entre clones das propriedades físicas e mecânicas da madeira de híbridos de Eucalyptus. Scientia Forestalis, n. 64, p. 33-47, 2003.

Fundação Centro Tecnológico de Minas Gerais. Uso da madeira para fins energéticos. Belo Horizonte, 1980. 158 p. (Série de publicações técnicas, 001).

Gomes, E. O. \& Oliveira, J. B. Teoria da carbonização da madeira. In: Penedo, W. R. (Ed.). Uso da madeira para fins energéticos. Belo Horizonte: CETEC, 1980. v. 1. p. 27-42.

Hein, P. R. G. et al. Estimativa da resistência e da elasticidade à compressão paralela às fibras da madeira de Eucalyptus grandis e E. urophylla usando a espectroscopia no infravermelho próximo. Scientia Forestalis, v. 37, n. 82, p. 119-129, 2009.
Indústria Brasileira de Árvores. IBÁ: Indústria Brasileira de Árvores. Brasília, 2015. 80 p. Relatório Ibá 2015.

Mendes, M. G. et al. Propriedades e controle da qualidade do carvão vegetal. In: Fundação Centro Tecnológico de Minas Gerais. Produção e utilização de carvão vegetal. Belo Horizonte, 1982. p. 77-89.

Moura, M. C. O. Variações em características de crescimento e da madeira em clones de Eucalyptus. 2000. 63 f. Tese (Mestrado em Ciência e Tecnologia da Madeira) - Universidade Federal de Lavras, Lavras.

Moutinho, V. H. P. Influência da variabilidade dimensional e da densidade da madeira de Eucalyptus sp. e Corymbia sp. na qualidade do carvão. 2013. 164 f. Tese (Doutorado em Recursos Florestais) - Escola Superior de Agricultura "Luiz de Queiroz", Universidade de São Paulo, Piracicaba.

Neves, T. A. et al. Avaliação de clones de Eucalyptus em diferentes locais visando à produção de carvão vegetal. Pesquisa Florestal Brasileira, v. 31, n. 68, p. 319-330, 2011. DOI: 10.4336/2011. pfb.31.68.319.

Protásio, T. P. et al. Mass and energy balance of the carbonization of babassu nutshell as affected by temperature. Pesquisa Agropecuária Brasileira, v. 49, n. 3, p. 189-196, 2014a. DOI: 10.1590/S0100204X2014000300005

Protásio, T. P. et al. Potencial siderúrgico e energético do carvão vegetal de clones de Eucalyptus spp. aos 42 meses de idade. Pesquisa Florestal Brasileira, v. 33, n. 74, p. 137-149, 2013. DOI: 10.4336/2013.pfb.33.74.448.

Protásio, T. P. et al. Qualidade da madeira e do carvão vegetal oriundos de floresta plantada em Minas Gerais. Pesquisa Florestal Brasileira, v. 34, n. 78, p. 111-123, 2014b. DOI: 10.4336/2014. pfb.34.78.657.

Protásio, T. P. et al. Quality and energetic evaluation of the charcoal made of babassu nut residues used in the steel industry. Ciência e Agrotecnologia, v. 38, n. 5, p. 435-444, 2014c. DOI: 10.1590/ S1413-70542014000500003.

Reis, A. A. et al. Composição da madeira e do carvão vegetal de Eucalyptus urophylla em diferentes locais de plantio. Pesquisa Florestal Brasileira, v. 32, n. 71, p. 277-290, 2012a. DOI: 10.4336/2012.pfb.32.71.277.

Reis, A. A. et al. Efeito de local e espaçamento na qualidade do carvão vegetal de um clone de Eucalyptus urophylla S. T. Blake. Floresta e Ambiente, v. 19, n. 4, p. 497-505, 2012b. DOI: 10.4322/ floram.2012.055.

Trugilho, P. F. et al. Avaliação de clones de Eucalyptus para a produção de carvão vegetal. Cerne, v. 7, n. 2, p. 104-114, 2001.

Vale, A. T. et al. Potencial energético da biomassa e carvão vegetal do epicarpo e da torta do pinhão-manso (Jatropha curcas). Cerne, v. 17 , n. 2, p. 267-273, 2011.

Vieira, R. da S. Propriedades mecânicas da madeira de clones de Eucalyptus e do carvão produzido entre $350^{\circ} \mathrm{C}$ e $\mathbf{9 0 0}^{\circ} \mathrm{C}$. 2009. $80 \mathrm{f}$. Tese (Doutorado em Ciência e Tecnologia da Madeira) - Universidade Federal de Lavras, Lavras. 
